\title{
Evaluación del nivel de satisfacción del estudiante respecto al servicio educativo bajo el enfoque del modelo HEdPERF en las universidades públicas que integran la Alianza Estratégica de la Universidad Peruana y que implementaron el mecanismo de licenciamiento ${ }^{1}$
}

Rosa Carolina Alvarez Tay ${ }^{2}$

Recibido: 02/05/2020 Aceptado: 19/01/2021 Publicado: 26/07/2021

\begin{abstract}
RESUMEN
El presente artículo tuvo como objetivo establecer el nivel de influencia del modelo HEdPERF (Higher Education Performance) en la satisfacción de los estudiantes, establecer el grado de influencia de cada una de las seis dimensiones que componen el Modelo HEdPERF en la satisfacción de los estudiantes y determinar cuál o cuáles de las seis dimensiones evaluadas del Modelo HEdPERF presentaban un nivel adecuado de la calidad percibida. La muestra del estudio estuvo comprendida por 1191 estudiantes pertenecientes a las tres universidades públicas socias de la Alianza Estratégica de la Universidad Peruana. La investigación empleó el diseño no experimental de nivel correlacional, y para el análisis de los datos se utilizaron las pruebas estadísticas no paramétricas, tales como la prueba de chi cuadrado, la prueba de correlación de Spearman y la prueba de signos de Wilcoxon.
\end{abstract}

Palabras clave: calidad del servicio; educación superior; modelo HEdPERF; aseguramiento de la calidad del servicio y satisfacción del estudiante.

\section{INTRODUCCIÓN}

En su "Informe sobre la educación superior en América Latina y el Caribe 2000-2005", el Instituto Internacional para la Educación Superior en América Latina y el Caribe (IESALC) señala que, bajo el discurso de defensa de la autonomía de las universidades y ante el descrédito total del Estado como proveedor eficaz de servicios públicos, se dispuso que los estudiantes (clientes) serían quienes asumirían la responsabilidad de seleccionar las opciones de mejor calidad educativa y que, a su vez, el mercado de las remuneraciones sancionaría los salarios de sus egresados de acuerdo al valor que el mercado de los demandantes atribuyese a sus títulos e instituciones de procedencia (Instituto Internacional para la Educación Superior en América Latina y el Caribe, 2015).

Aunado a ello, el Perú experimentó un proceso de rápido incremento de matrículas en el ámbito universitario durante las dos últimas décadas, lo cual, según la Dirección General de Educación Superior Universitaria, se vio reflejado en las cifras durante los años 1996 al 2012, donde la tasa de crecimiento fue de 2.5 veces más que los años anteriores, como resultado de la creación de 82 nuevas universidades (23 públicas y 59 privadas). Este incremento, sumado a una débil o casi nula planificación y a la ausencia de políticas sectoriales de aseguramiento de la calidad, conllevó al Estado Peruano a reconocer en su momento que "La educación superior universitaria no forma ciudadanos con altas capacidades para el ejercicio profesional competente y la producción de conocimiento de alto valor social" (D.S. $\mathrm{N}^{\circ}$ 016-2015-MINEDU, 2015).

\footnotetext{
Es un mecanismo que permite al Estado peruano asegurar que las instituciones que entran al mercado a proveer servicios de educación superior cumplan con exigencias mínimas de calidad.

Bachiller en Ciencias con mención en Ingeniería en Gestión Empresarial por la Universidad Nacional Agraria La Molina, y especialista en Gestión de Proyectos de Inversión Pública. Actualmente es consultora independiente (Lima, Perú)

ORCID: https://orcid.org/0000-0002-6378-0142

Autor de correspondencia: carolinaalvareztay@gmail.com
} 


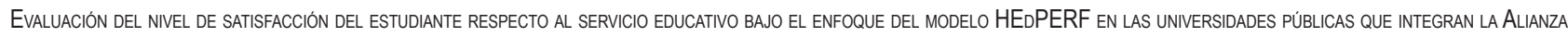
Estratégica de la Universidad Peruana y que implementaron el meCanismo de licenciamiento

De ahí que el Estado peruano, ante la urgente necesidad de reformas del sistema de educación universitaria durante el último decenio (2009-2019), por medio del Ministerio de Educación y sus organismos públicos adscritos, ha desplegado esfuerzos a través de diversos mecanismos como garantía de la calidad educativa que anteponen al estudiante como centro (D.S. No 016-2015-MINEDU, 2015), entre los cuales destacan el licenciamiento institucional, la acreditación de programas de estudios y universidades, y la certificación profesional; siendo el primero obligatorio y los dos mecanismos restantes, voluntarios.

Al respecto, las universidades públicas socias que pertenecen a la Alianza Estratégica de la Universidad Peruana ${ }^{3}$ obtuvieron su licenciamiento institucional por un plazo de hasta diez años, que entró en vigencia desde la fecha de su expedición en el siguiente orden cronológico: La Universidad Nacional Agraria La Molina (UNALM) en marzo del 2017 a través de la Resolución del Consejo Directivo $\mathrm{N}^{\circ}$ 011-2017-SUNEDU/CD (2017); la Universidad Nacional de Ingeniería (UNI) en noviembre del 2017 a través de la Resolución del Consejo Directivo N. 073-2017-SUNEDU/CD (2017); y, finalmente, la Universidad Nacional Mayor de San Marcos (UNMSM) en abril del 2018 a través de la Resolución del Consejo Directivo N ${ }^{\circ}$ 036-2018-SUNEDU/ CD (2018). De lo anteriormente expuesto, se concluye que a la fecha de la presente investigación ha trascurrido un periodo de tiempo de entre 2 a 3 años desde que estas universidades obtuvieron su licenciamiento, mecanismo que evalúa las Condiciones Básicas de Calidad (CBC).

En ese sentido, los problemas que pretende resolver la presente investigación se enuncian a continuación en forma de interrogante: (1) ¿En qué medida la aplicación del Modelo HEdPERF, enfocado en la calidad percibida de los servicios educativos, influye significativamente en la satisfacción de los estudiantes?, (2) ¿en qué medida cada una de las dimensiones que componen el Modelo HEdPERF influye significativamente en la satisfacción de los estudiantes? y (3) ¿cuál o cuáles de las dimensiones evaluadas del Modelo HedPerf presentan un nivel adecuado de la calidad percibida? De ahí que los objetivos de este artículo se expresen de la siguiente manera: (1) Establecer el nivel de influencia del Modelo HEdPERF (Higher Education Performance), enfocado en la calidad percibida de

3 Fue suscrita con fecha del 19 de marzo del 2012, entre las tres universidades nacionales y centenarias (UNALM, UNMSM y UNI) con la finalidad de contribuir a la recuperación, modernización y desarrollo de la universidad pública en el Perú. los servicios, en la satisfacción de los estudiantes, (2) establecer el grado de influencia de cada una de las seis dimensiones que componen el Modelo HEdPERF en la satisfacción de los estudiantes y (3) determinar cuál o cuáles de las seis dimensiones evaluadas del Modelo HEdPERF presentan un nivel adecuado de la calidad percibida.

Las hipótesis de este trabajo se formularon tomando como marco de referencia los problemas y objetivos presentados anteriormente; seguidamente, se muestran a detalle: (1) La calidad percibida de los servicios educativos del Modelo HedPerf influye significativamente en la satisfacción de los estudiantes, (2) las seis dimensiones que componen el Modelo HEdPERF influyen significativamente en la satisfacción de los estudiantes y (3) las dimensiones que componen el Modelo HEdPERF presentan un nivel adecuado de calidad percibida. De igual manera, estas hipótesis fueron contrastadas con cada una de las universidades públicas comprendidas en esta investigación a fin de realizar un parangón entre las mismas.

La principal contribución que el artículo pretende realizar está dirigida hacia las partes interesadas que componen el sistema peruano de educación universitaria, es decir, los estudiantes, los responsables de verificar el cumplimiento de la totalidad de las CBC y las autoridades universitarias encargadas de tomar decisiones de índole académica y no académica; esto, mediante la presentación en términos cualitativos y cuantitativos de la percepción de la calidad de los servicios educativos bajo el enfoque del modelo HEdPERF y su grado de influencia en la satisfacción de los estudiantes. Adicionalmente, la contribución del estudio como un aporte de conocimiento novedoso radica en la adaptación del idioma inglés al español de la escala del Modelo HEdPERF y su aplicación a la realidad de las universidades públicas integrantes de la Alianza Estratégica de la Universidad Peruana que implementaron el mecanismo de licenciamiento institucional.

Este estudio sienta sus bases sobre lo versado por los siguientes autores: Abdullah (2006), quien afirma que la evaluación del nivel de calidad, así como la comprensión de cómo las diversas dimensiones afectan la calidad general del servicio educativo, permitirá a las instituciones de educación superior diseñar eficientemente el proceso de entrega de servicios; Sunanto et al. (2007), quienes sugieren que las instituciones deben ver a los estudiantes como sus principales clientes y tratar de maximizar su satisfacción en función de los servicios educativos prestados; Spilimbergo (2009), quien sostiene 
que la capacidad de las universidades para promover un marco institucional de calidad, al ofrecer un entorno propicio para el diálogo democrático e intercambio de ideas, permite una formación integral que incluye el desarrollo de valores y habilidades interpersonales que se traducen en una mayor cohesión social y en el fortalecimiento democrático; Zineldin y Vasicheva (2014), quienes señalan que las mediciones de las percepciones de los estudiantes sobre la calidad del servicio ofrecido por una universidad pueden reflejar el nivel de satisfacción general de los estudiantes dentro de la institución; Govender et al. (2014), quienes indican que proporcionar servicios de calidad para las instituciones de educación superior es clave para diferenciarse de sus competidores y para garantizar la sostenibilidad durante un largo periodo; y finalmente, Nadim y Al Hinai (2016), quienes mencionan que la calidad en las instituciones de educación superior es uno de los aspectos más significativos de la creación de conocimiento, el desarrollo de recursos humanos y la fuerza social de cualquier país.

En referencia a otros estudios relacionados al presente artículo, los investigadores Abu Hasan et al. (2008) afirman que existe una relación positiva fuerte entre la calidad percibida y la satisfacción del estudiante; sumado a ello, Gallifa y Batallé (2010) confirman que si la prestación de servicios educativos no es dinámica, con el paso del tiempo el servicio se percibe como uno de baja calidad; por otro lado, Silva et al. (2017) revisaron sistemáticamente literatura académica para evaluar la pertinencia del Modelo HEdPERF como escala para medir la calidad de servicio percibida por los estudiantes en las instituciones de educación superior a nivel internacional, y concluyeron en que no es apropiado usar una escala genérica para todos los servicios, sino una escala específica para estimar cuantitativamente la calidad percibida en cada uno.

Finalmente, el marco teórico que soporta esta investigación se encuentra estructurado en tres ejes principales, como son:

1. Modelos de gestión de la calidad aplicados al entorno de instituciones de educación superior universitaria, para lo cual se llevó a cabo la revisión de otros artículos similares, encontrándose entre los modelos más destacados: (i) Modelo de gestión de calidad total (GCT), que se define como un sistema de garantías diseñado para dar cabida a múltiples partes interesadas, así como a los diversos y cambiantes roles de los estudiantes en el proceso educativo (Murad y Shastri, 2010); (ii) cuadro de mando integral (CMI), conceptualizado como un sistema de gestión simple, lógico, práctico y comprobado, que permite una adecuada respuesta a los desafíos modernos que enfrentan las instituciones de educación superior (Eftimov et al., 2016); (iii) modelo de la Fundación Europea para la Gestión de la Calidad (EFQM, por sus siglas en inglés), descrito como una evaluación sistemática que realiza una organización en todas sus áreas, comparándolas con el modelo de excelencia que le sirve de referencia; esto último permitiría a la alta dirección fijar planes de mejora basados en hechos objetivos y en una visión común de las metas a alcanzar y los medios a emplear (Del Campo et al., 2013); (iv) modelo ISO 9001:2015, ya que, en un contexto de crecientes tasas de inscripción, la estructura cambiante de la sociedad, los diferentes tipos de entrega de los programas educativos y las descripciones de puestos a gran escala, la aplicación de esta norma resulta de gran importancia, pues proporciona un conjunto de requisitos genéricos para implementar un sistema de gestión de calidad independientemente de las actividades que realice la organización (Hussein et al., 2017); (v) modelo ISO 21001: 2018, que se centra principalmente en la evaluación crítica y continua de las organizaciones educativas a fin de evaluar el grado de cumplimiento de los requisitos de los estudiantes y otros beneficiarios, de modo que se pueda mejorar el desempeño global de la organización (Organización Internacional de Normalización, 2018); y (vi) modelo de sistema integrado de aseguramiento de calidad (SIAC), que es considerado una buena práctica internacional y consta por lo general de los siguientes elementos: licenciamiento, acreditación, auditoría académica y sistemas de información (Pontificia Universidad Católica de Chile, 2011). Después del análisis de los modelos mencionados, se identificó que el SIAC es el que más se asemeja al contexto universitario peruano como garantía de la calidad educativa.

2. Modelos empleados para estudiar la calidad del servicio educativo universitario. Se realizó una revisión acuciosa de la literatura académica de los principales modelos utilizados en el ámbito universitario, tales como fueron: (i) Modelo del puntaje neto del promotor (PNP), el cual, según los autores Schmatz et al. (2015), consiste en una pregunta (la última pregunta de la encuesta) que evita términos como el de "satisfacción" o "calidad" por completo y más bien aborda algo que va incluso más allá de la satisfacción del cliente: la lealtad (la voluntad de hacer una inversión o un sacrificio personal para fortalecer una relación), lo 


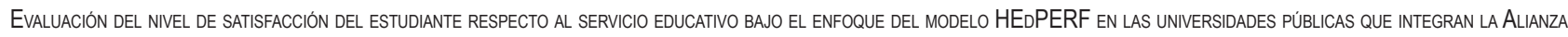
Estratégica de la Universidad Peruana y que implementaron el mecanismo de licenciamiento

que permite predecir los comportamientos reales de los clientes; (ii) modelo Kano, definido por Arefi et al. (2012) como un instrumento ampliamente utilizado para percibir la voz del cliente a fin de influenciar en la satisfacción del mismo; este modelo clasifica a los requerimientos del cliente en 6 categorías: elementos atractivos, elementos unidimensionales, elementos imprescindibles, elementos indiferentes, elementos inversos y elementos cuestionables; (iii) modelo SERVQUAL, conceptualizado por las autoras Cañón y Rubio (2018) como una escala de respuesta múltiple diseñada para comprender las expectativas de los clientes respecto a un servicio recibido a través de la evaluación de cinco dimensiones: tangibles, confiabilidad, capacidad de respuesta, aseguramiento y empatía; (iv) modelo SERVPERF, que, de acuerdo con los autores Maldonado y Moreta (2018), centra su atención en las percepciones de los clientes sobre la atención que reciben, suprimiendo las expectativas, y que además proporciona diversidad y variabilidad de acuerdo a la atención adecuada a los clientes; y (v) modelo HEdPERF , definido por su creador y autor Abdullah (2005) como una escala que mide la calidad percibida específicamente en el sector de educación superior, la misma que se encuentra compuesta por 6 dimensiones y 41 preguntas. Por lo anteriormente expuesto y apelando a los criterios de conveniencia y pertinencia para la presente investigación, se decidió aplicar en el estudio, la escala propuesta por el Modelo HEdPERF.

3. Bases legales en el contexto del aseguramiento de la calidad educativa superior universitaria peruana, conformadas principalmente por: (1) La Nueva Ley Universitaria (Ley $N^{\circ}$. 30220, 2014), que trajo consigo profundos cambios como la creación de la Superintendencia Nacional de Educación Universitaria (SUNEDU), que tiene a su cargo garantizar el cumplimiento de las Condiciones Básicas de Calidad (CBC) inmersas en el proceso de licenciamiento institucional, y que precisa al proceso de acreditación como voluntario salvo excepciones; y (2) la Política de Aseguramiento de la Calidad de la Educación Superior Universitaria, que establece cinco principios (autonomía y rectoría responsable, estudiante como centro, inclusión y equidad, calidad y excelencia académica, y desarrollo del país) y cuatros pilares (información confiable y oportuna, fomento para mejorar el desempeño, acreditación para la mejora continua, y licenciamiento como garantía de condiciones básicas de calidad) en el marco del sistema universitario peruano.

\section{METODOLOGÍA}

El diseño de la investigación fue de corte transeccional, no experimental y correlacional. Seguidamente, se describe a detalle:

- Es transeccional, es decir, que los datos fueron recolectados en un solo momento (Ciclo académico 2019 - II) y su propósito fue describir las variables y analizar su incidencia e interrelación en un momento dado.

- Es no experimental, ya que la variable dependiente (satisfacción del estudiante) en el estudio ocurrió y no fue posible manipularla, es decir, no se tuvo control directo sobre dicha variable, puesto que ya había sucedido, al igual que sus efectos. Al realizar una investigación no experimental, se observó el fenómeno tal y como se suscitó en su contexto natural (universidades públicas socias que pertenecen a la Alianza Estratégica de la Universidad Peruana) para su posterior análisis.

- Es correlacional, puesto que se describieron las relaciones entre las variables independientes (calidad percibida de los servicios educativos y subvariables) y la variable dependiente (satisfacción del estudiante); de igual manera, se estableció su dimensión y dirección.

La unidad de análisis estuvo compuesta por estudiantes matriculados de pregrado y posgrado, pertenecientes a los campus que concentran la mayor cantidad de programas de estudios en las tres universidades públicas socias que integran la Alianza Estratégica de la Universidad Peruana (Ver Tabla 1).

Tabla 1. Localización de las universidades del estudio.

\begin{tabular}{|l|l|l|}
\hline \multicolumn{1}{|c|}{ Universidad } & \multicolumn{1}{c|}{ Dirección } & \multicolumn{1}{c|}{ Distrito } \\
\hline Universidad Nacional Mayor de San Marcos & Calle German Amezaga N 375 & Cercado de Lima \\
\hline Universidad Nacional Agraria La Molina & Avenida La Molina S/N & La Molina \\
\hline Universidad Nacional de Ingeniería & Avenida Túpac Amaru N ${ }^{\circ} 210$ & Rímac \\
\hline
\end{tabular}

Fuente: Elaboración propia. 
Las características de la unidad de análisis con respecto al género fueron: $66 \%$ de varones, $31 \%$ de mujeres y $3 \%$ de otros. Con relación al intervalo de edades de los estudiantes, se obtuvo que el $14 \%$ se encontraba entre las edades desde los 14 hasta los 18 años, $58 \%$ desde los 19 hasta los 22 años, 22\% desde los 23 hasta los 26 años y $6 \%$ desde los 27 hasta los 58 años. Con respecto al nivel académico, el $96 \%$ pertenecía a pregrado y el $4 \%$ pertenecía a posgrado. En referencia al ciclo académico, el 54\% declaró encontrarse entre el primer y cuarto ciclo, el 33\% pertenecía desde el quinto hasta el octavo ciclo y el $13 \%$ se encontraba entre el noveno y décimo segundo ciclo. Finalmente, con respecto a las áreas de conocimiento, el $71 \%$ pertenecía al área de las ingenierías; el 13\% al área de las Ciencias Básicas; el 7\% del área de las Humanidades, Ciencias Jurídicas y Sociales; el 6\% al área de Ciencias Económicas y de Gestión; y el 2\% del área de las Ciencias de la Salud.

El tamaño total de la muestra de la investigación, según se aprecia en la Tabla 2, fue de 1191 alumnos matriculados y este se calculó utilizando los valores históricos del número de alumnos matriculados entre los años 2014 y 2018 con la finalidad de proyectar el promedio de alumnos matriculados para el año 2019; posteriormente, se empleó la fórmula para el cálculo del tamaño de muestra para una población finita, con un nivel de confianza del $95 \%$, y se obtuvo para cada universidad del estudio la siguiente distribución:

La selección de la muestra se realizó de forma aleatoria y estratificada proporcional, aplicando este último criterio según la universidad de procedencia. De igual forma, se aplicaron otros criterios, tales como los que se detallan a continuación:

Criterio de exclusión: Alumnos de pregrado y posgrado que no se matricularon en el ciclo académico 2019-II o que se no se encontraban, durante el estudio de campo, dentro de los campus que concentran la mayor población estudiantil en las 3 universidades públicas socias que integran la Alianza Estratégica de la Universidad Peruana.

Criterio de eliminación: Se eliminaron las encuestas de aquellos estudiantes que no completaron alguno de los ítems que componen el instrumento o que duplicaron su respuesta en alguno de los ítems solicitados o que tuvieron patrones inusuales de respuesta, tales como elegir la misma alternativa en casi todos los ítems.

La recolección de los datos se desarrolló a través de una encuesta en el marco de trabajo del Modelo HEdPERF, la cual estuvo compuesta por 41 preguntas (ítems) de fácil comprensión, breves y concisas. Respecto al análisis e interpretación de la información, se utilizaron los paquetes informáticos de procesamiento y análisis estadístico MINITAB versión 17 y SPSS versión 25. Asimismo, se emplearon otros softwares complementarios para la extracción y la visualización (tablas y gráficos) de los datos, como fueron el Microsoft Excel y Microsoft Power Point 2016.

\section{RESULTADOS}

En la Tabla 3, se muestra a detalle la descripción de las 6 dimensiones que propone el Modelo HEdPERF, cuya correcta comprensión permitió la interpretación de los resultados obtenidos en el presente estudio y facilitó la discusión posterior de las implicancias de este artículo.

Por otro lado, como parte de los actos preparatorios a la aplicación del instrumento de medición (encuesta) a la muestra total y al desarrollo de la contrastación de las hipótesis de investigación, se desarrolló una prueba piloto con una submuestra compuesta por 53 estudiantes provenientes de las 3 universidades públicas socias de la Alianza Estratégica de la Universidad Peruana (17 de la UNALM, 19 de la UNI y 17 de la UNMSM) con el propósito de evaluar la confiabilidad y validez del instrumento de medición.

Tabla 2. Tamaño de muestra del estudio.

\begin{tabular}{|l|c|c|}
\hline \multicolumn{1}{|c|}{ Universidad de procedencia } & Tamaño de la población & Tamaño de muestra \\
\hline Universidad Nacional Agraria La Molina & 13159 & 384 \\
\hline Universidad Nacional Mayor de San Marcos & 42787 & 392 \\
\hline Universidad Nacional de Ingeniería & 22888 & 415 \\
\hline Total & $\mathbf{7 8 8 3 4}$ & 1191 \\
\hline
\end{tabular}

Fuente: Elaboración propia. 


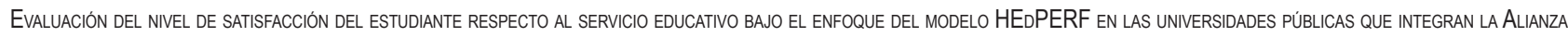
Estratégica de la Universidad Peruana y que implementaron el meCanismo de licenciamiento

La consistencia interna de los ítems que componen cada una de las 6 dimensiones de la escala del Modelo HEdPERF confirmó que la encuesta era confiable en la medida en que sus dimensiones obtuvieron coeficientes de alfa de Cronbach con valores que oscilan entre 0.780 y 0.924 (ver Tabla 4).

La validación del contenido de la encuesta se realizó a través de la técnica de juicio de expertos; para ello se contó con la participación de 3 docentes universitarios, los cuales son especialistas en los campos de evaluación de pruebas psicométricas, evaluación de tesis de posgrado y gestión de calidad de servicios educativos. En consecuencia, los expertos a través de 5 criterios de evaluación (congruencia de los ítems, amplitud del contenido, redacción de los ítems, claridad y precisión, y pertinencia) manifestaron sus opiniones, que fluctuaron entre las valoraciones de aceptable y excelente (ver Tabla 5), lo que dio la confianza para validar el contenido del instrumento de medición.

La comprobación del supuesto de la normalidad de la distribución de los datos de las variables (a) calidad percibida del servicio educativo y sus subvariables (dimensiones del modelo HEdPERF) y (b) satisfacción del estudiante, se realizó a través de las pruebas de normalidad Kolmogorov Smirnov (SPSS versión 25) y Anderson Darling (Minitab versión 17). Se concluyó que presentan distribuciones

Tabla 3. Descripción cualitativa de las dimensiones del Modelo HEdPERF.

\begin{tabular}{|l|l|}
\hline \multicolumn{1}{|c|}{ Dimensión } & \multicolumn{1}{c|}{ Descripción } \\
\hline Aspectos académicos & Elementos por los que la plana docente es completamente responsable. \\
\hline Aspectos no académicos & $\begin{array}{l}\text { Elementos que son esenciales para permitir que el estudiante cumpla con sus requisitos de estudio. } \\
\text { Se refiere a las tareas realizadas por el personal administrativo. }\end{array}$ \\
\hline Reputación & $\begin{array}{l}\text { Elementos que sugieren la importancia en las instituciones de educación superior de proyectar la } \\
\text { imagen profesional. }\end{array}$ \\
\hline Acceso & Elementos relacionados a la accesibilidad, facilidad de contacto, disponibilidad y conveniencia. \\
\hline Programas & $\begin{array}{l}\text { Elementos que enfatizan la importancia de proveer de un amplio rango de programas con elevada } \\
\text { reputación, con estructuras flexibles y planes de estudios. }\end{array}$ \\
\hline Entendimiento & $\begin{array}{l}\text { Elementos referidos al entendimiento específico de las necesidades de los estudiantes en términos de } \\
\text { consejería y salud. }\end{array}$ \\
\hline
\end{tabular}

Fuente: Adaptado de Silva et al. (2017).

Tabla 4. Resultados de la consistencia interna.

\begin{tabular}{|l|c|c|c|}
\hline \multicolumn{1}{|c|}{ Dimensiones } & Alfa de Cronbach & Número de ítems & Criterios \\
\hline Aspectos académicos & 0.893 & 9 & Alta confiabilidad \\
\hline Aspectos no académicos & 0.924 & 12 & Confiabilidad excelente \\
\hline Reputación & 0.915 & 8 & Confiabilidad excelente \\
\hline Acceso & 0.868 & 7 & Alta confiabilidad \\
\hline Programas & 0.846 & 2 & Alta confiabilidad \\
\hline Entendimiento & 0.780 & 2 & Alta confiabilidad \\
\hline
\end{tabular}

Fuente: Elaboración propia.

Tabla 5. Resultados según los criterios de evaluación por parte de los expertos participantes.

\begin{tabular}{|c|c|c|c|c|c|}
\hline & 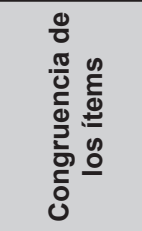 & 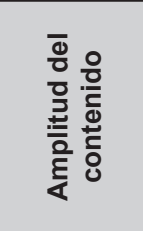 & 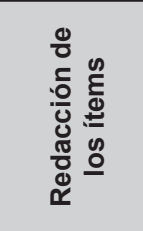 & 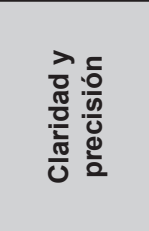 & 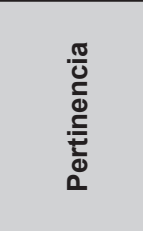 \\
\hline Experto n. ${ }^{\circ} 01$ & Excelente & Bueno & Bueno & Bueno & Excelente \\
\hline Experto $n .^{\circ} 02$ & Bueno & Bueno & Bueno & Excelente & Bueno \\
\hline Experto ${ }^{\circ}{ }^{\circ} 03$ & Aceptable & Aceptable & Aceptable & Aceptable & Aceptable \\
\hline
\end{tabular}

Fuente: Elaboración propia 
no normales, puesto que poseen un $p$-valor menor a 0.05 (valor de $\alpha$ ), con un intervalo de confianza del $95 \%$ y, por consiguiente, se procedieron a contrastar las hipótesis de investigación mediante pruebas estadísticas no paramétricas (ver Tabla 6).

Para la contrastación de las hipótesis del artículo, se emplearon las siguientes pruebas estadísticas no paramétricas: (1) la prueba de chi cuadrado, que permite determinar la asociación o independencia de dos variables cualitativas a través del empleo de tablas de contingencia donde se resumen los datos categóricos por filas y columnas; (2) la prueba de rangos con signos de Wilcoxon, que según los autores Juárez, López y Villatoro (2014), se emplea para comparar la mediana (posición central en un conjunto de datos ordenados) de dos muestras relacionadas y determinar si existen diferencias entre ellas; y (3) la prueba de correlación de Spearman, que posibilita conocer el grado de asociación entre dos variables a través de la Rho de Spearman que a su vez determina la dependencia o independencia de dos variables aleatorias, así como qué tan fuerte es la relación entre las variables y si la relación entre dichas variables es positiva, nula o negativa (Elorza y Medina, 1999).

Los resultados para la primera hipótesis de investigación se muestran en la Tabla 7 , donde se observa que los estudiantes que calificaron la calidad percibida de los servicios como "alta" fueron los que más satisfechos estuvieron con dichos servicios educativos.

Adicionalmente, para la primera hipótesis de investigación se aprecia en la Tabla 8 (tabla de contingencia), donde los resultados de la prueba chi cuadrado tienen un nivel de confianza del $95 \%$, que el valor calculado del chi cuadrado de Pearson (242.419) fue mayor al valor crítico del chi cuadrado (9.4877). Asimismo, se obtuvo que el coeficiente de significancia $(p=0.000)$ fue menor a $0.05(p<0.05)$, lo cual permitió aceptar la hipótesis de investigación.

Por lo tanto, se puede afirmar que la percepción de calidad de los servicios educativos del modelo

Tabla 6. Resumen de las Pruebas de Normalidad.

\begin{tabular}{|l|c|c|c|}
\hline \multirow{2}{*}{\multicolumn{1}{|c|}{ Dimensiones }} & \multicolumn{2}{c|}{ Kolmogorov - Smirnov } & Anderson Darling \\
\cline { 2 - 4 } & Estadístico de prueba & Significancia asintótica (Bilateral) & $p$-valor \\
\hline Aspectos académicos & 0.053 & 0.000 & $p$-valor $<0.005$ \\
\hline Aspectos no académicos & 0.051 & 0.000 & $p$-valor $<0.005$ \\
\hline Reputación & 0.050 & 0.000 & $p$-valor $<0.005$ \\
\hline Acceso & 0.050 & 0.000 & $p$-valor $<0.005$ \\
\hline Programas & 0.124 & 0.000 & $p$-valor $<0.005$ \\
\hline Entendimiento & 0.104 & 0.000 & $p$-valor $<0.005$ \\
\hline Satisfacción del servicio & 0.337 & 0.000 & $p$-valor $<0.005$ \\
\hline
\end{tabular}

Fuente: Elaboración propia.

Tabla 7. Nivel de influencia de la percepción de la calidad de los servicios educativos y la satisfacción de los estudiantes.

\begin{tabular}{|c|c|c|c|c|c|c|}
\hline & & & \multicolumn{3}{|c|}{ Satisfacción del Estudiante } & \multirow[b]{2}{*}{ Total } \\
\hline & & & Decepcionado & Indiferente & Encantado & \\
\hline \multirow{6}{*}{$\begin{array}{l}\frac{\pi}{0} \\
\frac{0}{0} \\
\frac{0}{0} \\
0 \\
0 \\
0 \\
\frac{\pi}{0} \\
\frac{\pi}{0} \\
\frac{\pi}{\sqrt{0}}\end{array}$} & \multirow[t]{2}{*}{ Baja } & Observado & 18.0 & 12.0 & 5.0 & 35.0 \\
\hline & & Esperado & 4.7 & 8.8 & 21.5 & 35.0 \\
\hline & \multirow[t]{2}{*}{ Media } & Observado & 70.0 & 96.0 & 50.0 & 216.0 \\
\hline & & Esperado & 28.8 & 54.6 & 132.6 & 216.0 \\
\hline & \multirow[t]{2}{*}{ Alta } & Observado & 71.0 & 193.0 & 676.0 & 940.0 \\
\hline & & Esperado & 125.5 & 237.6 & 576.9 & 940.0 \\
\hline & \multirow[t]{2}{*}{ Total } & Observado & 159.0 & 301.0 & 731.0 & 1191.0 \\
\hline & & Esperado & 159.0 & 301.0 & 731.0 & 1191.0 \\
\hline
\end{tabular}

Fuente: Elaboración propia. 


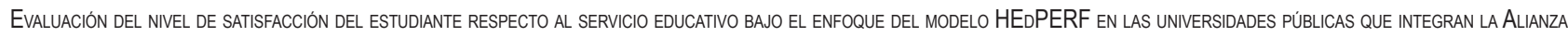
Estratégica de la Universidad Peruana y que implementaron el mecanismo de licenciamiento

HEdPERF influye o impacta en la satisfacción de los estudiantes que pertenecen a las tres universidades públicas socias de la Alianza Estratégica de la Universidad Peruana.

De igual manera, para la contrastación de la segunda hipótesis de investigación, mediante la cual se trató de probar que cada una de las dimensiones del Modelo HedPerf influye en la satisfacción del estudiante, se comprobó a través la prueba no paramétrica chi cuadrado que estas seis dimensiones influían en la satisfacción del estudiante (ver Tabla 9). Por otra parte, se realizó la comprobación de la influencia de las seis dimensiones en la satisfacción de los estudiantes según su casa de estudio de procedencia (UNALM, UNMSM y UNI), concluyéndose de igual manera que existe una relación de dependencia.
Luego de haberse comprobado en la segunda hipótesis que existía dependencia entre las variables calidad percibida de los servicios educativos y satisfacción del estudiante, al igual que existía dependencia entre cada una de las seis dimensiones del Modelo HEdPERF y la variable satisfacción del estudiante mediante la prueba de chi cuadrado, se procedió a verificar el tipo de asociación a través de la prueba no paramétrica correlación de Spearman, cuyos resultados mostraron una correlación positiva moderada para las dimensiones aspectos académicos, aspectos no académicos, programas y entendimiento; es decir, que sus valores se encontraban entre 0.11 y 0.50 ; mientras que, para las dimensiones reputación y acceso, se determinó que existía una correlación positiva considerable al encontrase entre los valores de 0.51 y 0.75 (ver Tabla 10).

Tabla 8. Prueba chi cuadrado para la percepción de la calidad de los servicios educativos y la satisfacción de los estudiantes.

\begin{tabular}{|l|c|c|c|}
\hline \multicolumn{1}{|c|}{ Estadísticos } & Valor & gl & Significación asintótica (bilateral) \\
\hline Chi cuadrado de Pearson & 242.419 & 4 & 0.000 \\
\hline Razón de verosimilitud & 229.4815 & 4 & 0.000 \\
\hline N de casos válidos & 1191 & & \\
\hline
\end{tabular}

Fuente: Elaboración propia.

Tabla 9. Prueba chi cuadrado de las seis dimensiones del Modelo HEdPERF y la variable satisfacción del estudiante.

\begin{tabular}{|l|l|c|l|}
\hline $\mathbf{N}^{\circ}$ & $\begin{array}{c}\text { Dimensiones del modelo } \\
\text { HEdPERF }\end{array}$ & $\begin{array}{c}\text { Valor de } \boldsymbol{\alpha} \text { versus } \\
\boldsymbol{p} \text {-valor }\end{array}$ & \multicolumn{1}{|c|}{ Resultado de la prueba chi cuadrado } \\
\hline 1 & Aspectos académicos & $0.000<0.05$ & $\begin{array}{l}\text { La dimensión "aspectos académicos" influye en la satisfacción del } \\
\text { estudiante }\end{array}$ \\
\hline 2 & Aspectos no académicos & $0.000<0.05$ & $\begin{array}{l}\text { La dimensión "aspectos no académicos" influye en la satisfacción del } \\
\text { estudiante }\end{array}$ \\
\hline 3 & Reputación & $0.000<0.05$ & La dimensión "reputación" influye en la satisfacción del estudiante \\
\hline 4 & Acceso & $0.000<0.05$ & La dimensión "acceso" influye en la satisfacción del estudiante \\
\hline 5 & Programas & $0.000<0.05$ & La dimensión "programas" influye en la satisfacción del estudiante \\
\hline 6 & Entendimiento & $0.000<0.05$ & La dimensión "entendimiento" influye en la satisfacción del estudiante \\
\hline
\end{tabular}

Fuente: Elaboración propia.

Tabla 10. Prueba de Correlación de Spearman de las seis dimensiones del Modelo HEdPERF y la variable satisfacción del estudiante.

\begin{tabular}{|l|l|c|l|}
\hline $\mathbf{N}^{\circ}$ & Dimensiones & Coeficiente de correlación (Rho) & Tipo de correlación \\
\hline 1 & Aspectos académicos & 0.411 & Correlación positiva media o moderada \\
\hline 2 & Aspectos no académicos & 0.487 & Correlación positiva media o moderada \\
\hline 3 & Reputación & 0.528 & Correlación positiva considerable \\
\hline 4 & Acceso & 0.526 & Correlación positiva considerable \\
\hline 5 & Programas & 0.464 & Correlación positiva media o moderada \\
\hline 6 & Entendimiento & 0.478 & Correlación positiva media o moderada \\
\hline
\end{tabular}

Fuente: Elaboración propia. 
Además de ello, se realizó el mismo análisis discriminando los resultados según la casa de estudios de procedencia. De ahí se obtuvo que para los estudiantes de la UNALM, las dimensiones que presentaron una correlación positiva moderada fueron: aspectos académicos, aspectos no académicos, reputación, programas y entendimiento; mientras que la única dimensión que obtuvo una correlación positiva considerable fue la dimensión acceso. Con respecto a la UNMSM, se tuvo a las dimensiones aspectos académicos y entendimiento con una correlación positiva moderada; mientras que las demás dimensiones obtuvieron una correlación positiva considerable. Por último, la UNI obtuvo una correlación positiva moderada para las seis dimensiones evaluadas.

Del mismo modo, para comprobar la tercera hipótesis de la investigación, respecto a identificar cuál o cuáles de las seis dimensiones evaluadas del Modelo HEdPERF presentaban un alto nivel de calidad percibida, es decir, entre las categorías o valoraciones de "Muy Bueno" y "Excelente", se aplicó la prueba estadística no paramétrica de Wilcoxon, comparando la mediana calculada respecto a una mediana hipotética. Se obtuvo que 4 de las 6 dimensiones evaluadas (aspectos académicos, aspectos no académicos, acceso y entendimiento) no poseen un alto nivel calidad percibida; en contraste, las 2 dimensiones restantes (reputación y programas) obtuvieron altos niveles de calidad percibida (ver Tabla 11).

Por otra parte, se consideró conveniente evaluar la tercera hipótesis de investigación de manera específica de acuerdo a la universidad de procedencia de los estudiantes. Se obtuvo que para la Universidad Nacional Agraria La Molina (UNALM), las dimensiones que presentaron un alto nivel de calidad percibida fueron aspectos académicos, reputación, acceso, programas y entendimiento; en lo que se refiere a la Universidad Nacional Mayor de San Marcos (UNMSM), solo la dimensión programas obtuvo un alto nivel de calidad percibida; por último, para la Universidad Nacional de Ingeniería (UNI), ninguna de las dimensiones obtuvo niveles adecuados de calidad percibida.

\section{DISCUSIÓN}

La consistencia interna de los ítems que componen cada una de las seis dimensiones de la escala HEdPERF, demostró que la encuesta es confiable en la medida en que sus dimensiones obtuvieron coeficientes de alfa de Cronbach con valores que oscilan entre 0.780 y 0.924 . Esto último se condice con los resultados obtenidos por los autores Abdullah (2006) y Brochado (2009), quienes concluyeron que la escala HEdPERF es la que se ajusta mejor al contexto de la educación superior en comparación con otros instrumentos "clásicos" de medición como son el SERVQUAL y el SERVPERF.

En referencia a la verificación de la normalidad de la distribución de los datos de las variables calidad percibida del servicio educativo, dimensiones y satisfacción del estudiante, a través de las pruebas de normalidad Kolmogorov Smirnov y Anderson Darling, se concluyó que presentan distribuciones no normales. Es por esto que, para la contrastación de las tres hipótesis de investigación de este artículo, se vio conveniente aplicar estadísticos no paramétricos, confirmando lo señalado por la autora Jamieson (2004), quien indica que sólo se deben utilizar estadísticos no paramétricos para el análisis de los datos de la escala de Likert.

Mediante la primera hipótesis de investigación se demostró que existe una relación significativa y positiva entre la calidad percibida de los servicios educativos propuestos por el Modelo HEdPERF y la satisfacción del estudiante, tal y como se eviden-

Tabla 11. Prueba de Signos de Wilcoxon de las seis dimensiones respecto a una mediana hipotética.

\begin{tabular}{|c|l|c|l|}
\hline$N^{\circ}$ & $\begin{array}{c}\text { Dimensiones del modelo } \\
\text { HEdPERF }\end{array}$ & $\begin{array}{c}\text { p-valor versus } \\
\text { valor de } \alpha\end{array}$ & \multicolumn{1}{|c|}{ Resultado de la prueba de signos de Wilcoxon } \\
\hline 1 & Aspectos académicos & $0.9730>0.05$ & La dimensión "aspectos académicos" no posee alto nivel de calidad percibida. \\
\hline 2 & Aspectos no académicos & $1.0000>0.05$ & $\begin{array}{l}\text { La dimensión "aspectos no académicos" no posee un alto nivel de calidad } \\
\text { percibida. }\end{array}$ \\
\hline 3 & Reputación & $0.0000<0.05$ & La dimensión "reputación" posee un alto nivel de calidad percibida. \\
\hline 4 & Acceso & $1.0000>0.05$ & La dimensión "acceso" no posee un alto nivel de calidad percibida. \\
\hline 5 & Programas & $0.0000<0.05$ & La dimensión "programas" posee un alto nivel de calidad percibida. \\
\hline 6 & Entendimiento & $0.1000>0.05$ & La dimensión "entendimiento" no posee un alto nivel de calidad percibida. \\
\hline
\end{tabular}

Fuente: Elaboración propia. 


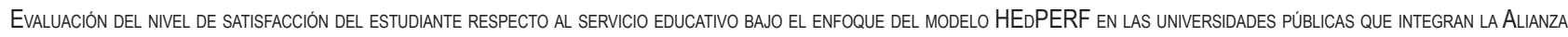
Estratégica de la Universidad Peruana y que implementaron el meCanismo de licenciamiento

ció en estudios realizados anteriormente (Nguyen et al., 2014; Banahene et al., 2018; Siti et al., 2020). Por otro lado, respecto a la segunda hipótesis de investigación, también se demostró que existe una relación significativa y positiva entre cada una de las seis dimensiones y la satisfacción del estudiante. Por último, para la tercera hipótesis de investigación se demostró que las dimensiones que poseen un alto nivel de calidad percibida fueron "reputación" y "programas"; esto último enriquece los resultados obtenidos por otros autores que plantearon hipótesis de investigación similares (Ali et al., 2016; Purwanto et al., 2020).

En relación a la generalización de la investigación, tomando como base los resultados de la muestra total (1191 estudiantes) y los resultados estratificados según la universidad de procedencia (384 estudiantes de la UNALM, 392 estudiantes de la UNMSM y 415 estudiantes de la UNI), se puede afirmar que en función al contexto en el cual la escala sea aplicada, es posible encontrar diferencias entre las seis dimensiones que componen el Modelo HEdPERF y su grado de influencia en la satisfacción del estudiante, así como, entre los niveles de calidad percibida por cada una de las dimensiones del modelo HEdPERF.

El presente trabajo de investigación supone un aporte al conocimiento teórico en el marco de trabajo de los diversos modelos que se vienen aplicando para medir la calidad del servicio en las instituciones de educación superior, debido a que, por primera vez, se aplicó la escala propuesta por el Modelo HEdPERF en un contexto de universidades públicas socias peruanas (Universidad Nacional Mayor de San Marcos, Universidad Nacional Agraria La Molina y Universidad Nacional de Ingeniería). Aunado a ello, la originalidad de este estudio radicó en la traducción del idioma inglés al español del instrumento de medición en cuestión, además del aporte de nuevas dimensiones para evaluar la calidad percibida de los servicios educativos, como fueron: aspectos académicos, aspectos no académicos, reputación, acceso, programas y entendimiento; según su autor y creador Abdullah (2005). Finalmente, esta investigación hace una contribución a la revisión sistémica de la literatura académica en materia de los modelos de gestión de la calidad educativa, así como de las principales escalas utilizadas para estudiar la calidad de los servicios educativos en las instituciones de educación superior y la normativa vigente en lo que respecta al aseguramiento de la calidad en la educación superior universitaria peruana.
Por otro lado, las implicancias prácticas del estudio permitieron presentar en términos cualitativos y cuantitativos la percepción de la calidad de los servicios educativos bajo el enfoque de Modelo HEdPERF y su grado de influencia en la satisfacción de los estudiantes en un contexto posterior a la implementación del mecanismo de licenciamiento institucional.

\section{CONCLUSIONES}

- La escala del Modelo HEdPERF ha demostrado ser un instrumento válido y confiable al haberse aplicado a alumnos de pre y posgrado de las universidades públicas socias que pertenecen a la Alianza Estratégica de la Universidad Peruana, por lo que puede ser utilizado en futuros estudios de investigación.

- Se logró comprobar la primera hipótesis de investigación, donde se concluyó que la calidad percibida propuesta por el Modelo HEdPERF influye significativamente en la satisfacción del estudiante, para ello se utilizaron las pruebas estadísticas no paramétricas como chi cuadrado y correlación de Spearman.

- Se logró comprobar la segunda hipótesis de investigación, por lo que se puede afirmar que el nivel de calidad percibida por cada una de las seis dimensiones del Modelo HEdPERF influye significativamente en la satisfacción del estudiante, para ello se utilizaron las pruebas estadísticas no paramétricas chi cuadrado y correlación de Spearman.

- Se logró comprobar la tercera hipótesis de investigación mediante la prueba de signos de Wilcoxon, determinándose los niveles de calidad percibida para cada una de las dimensiones del Modelo HEdPERF; obteniéndose que cuatro de las seis las dimensiones evaluadas (aspectos académicos, aspectos no académicos, acceso y entendimiento) no poseen un alto nivel de calidad percibida, mientras que, las dos dimensiones restantes (reputación y programas) obtuvieron altos niveles calidad percibida.

- Se realizó el análisis de la prueba de Wilcoxon para cada una de las universidades incluidas en el alcance de la presente investigación y se encontró que para la Universidad Nacional Agraria La Molina (UNALM), aquellas dimensiones que presentaban 
un alto nivel de calidad percibida fueron aspectos académicos, reputación, acceso, programas y entendimiento; para la Universidad Nacional Mayor de San Marcos (UNMSM), solo la dimensión programas obtuvo un alto nivel de calidad percibida; y para la Universidad Nacional de Ingeniería (UNI), ninguna de la dimensiones obtuvo altos niveles de calidad percibida. De lo anteriormente expuesto, se concluye que existen diferencias en los niveles de calidad percibida entre las dimensiones según la universidad de procedencia.

\section{REFERENCIAS BIBLIOGRÁFICAS}

[1] Abdullah, F. (2005). HEdPERF versus SERVPERF: The quest for ideal measuring instrument of service quality in higher education sector. Quality Assurance in Education, 13(4), 305-328. Recuperado de https://doi. org/10.1108/09684880510626584

[2] Abdullah, F. (2006). The development of HEdPERF: a new measuring instrument of service quality for the higher education sector. Internacional Journal of Consumer Studies, 30(6), 569-581. Recuperado de https://doi. org/10.1111/j.1470-6431.2005.00480.x

[3] Abu Hasan, H., Illias, A., Rahman, R., y Razack, M. (2008). Service Quality and Student Satisfaction: A Case Study at Private Higher Education Institutions. International Business Research, 1(3),163-175. Recuperado de https://doi.org/10.5539/ibr.v1n3p163

[4] Ali, F., Zhou, Y., Hussain, K., Nair, P., y Ravagan, N. (2016). Does higher education service quality effect student satisfaction, image and loyalty? A study of international students in Malaysian public universities. Quality Assurance in Education, 24(1), 70-94. Recuperado de https://doi.org/10.1108/QAE02-2014-0008

[5] Arefi, M., Heidari, M., Morkani, G., y Zandi, K. (2012). Application of Kano Model in Higher Education Quality Improvement: Study Master's Degree Program of Educational Psychology in State Universities of Tehran. World Applied Sciences Journal, 17 (3), 347353. Recuperado de https://www.idosi.org/ wasj/wasj17(3)12/12.pdf

[6] Banahene, S., Kraa, J., y Kasu, P. (2018). Impact of HEdPERF on Students' Satisfaction and Academic Performance in Ghanaian Universities; Mediating Role of Attitude towards
Learning. Open Journal of Social Sciences, 6(5), 96-119. Recuperado de https://doi. org/10.4236/jss.2018.65009

[7] Brochado, A. (2009). Comparing alternative instruments to measure service quality in higher education. Quality Assurance in Education, 17(2), 174 -190. Recuperado de https://doi. org/10.1108/09684880910951381

[8] Cañón, A., y Rubio, D. (2018). Importancia de la utilización del modelo SERVQUAL a partir de una revisión sistemática de la literatura en el periodo 2010-2016. (Trabajo de grado especialización). Fundación Universitaria de Ciencias de la Salud, Bogotá. Recuperado de https://repositorio.fucsalud.edu.co/ handle/001/669

[9] D.S. N $\mathrm{N}^{\circ}$ 016-2015-MINEDU. Aprueban la política de aseguramiento de la calidad de la educación superior universitaria. Diaria Oficial El Peruano (2015).

[10] Del Campo, M., Ferreiro, F, y Camino, M. (Julio de 2013). Una aplicación del modelo EFQM a la educaciónsuperior.XXIIJornadasdelaAsociación de la Economía de la Educación. Recuperado de http://2013.economicsofeducation. c o m / u s e r/pdf s e s i on e s/ 138 . pdf?PHPSESSID=evsluk1nq0k6noc2eipo8tlnt7

[11] Eftimov, L., Trpeski, P., Gockov, G., y Vasileva, V. (2016). Designing a Balanced Scorecard as Strategic Management System for Higher Education Institutions: A Case Study in Macedonia. Ekonomika, Journal for Economic Theory and Practice and Social Issues, 62(2), 29-48. Recuperado de https://doi.org/10.22004/ ag.econ. 288842

[12] Elorza, H., y Medina, J. (1999). Estadística para las ciencias sociales y del comportamiento. México D. F., México: Oxford University.

[13] Gallifa, J., y Batallé, P. (2010). Percepción de los estudiantes sobre la calidad del servicio en un sistema de educación superior con varios campus en España. Revista de Aseguramiento de la Calidad en la Educación, 18(2), 156 - 170. Recuperado de https://doi. org/10.1108/09684881011035367

[14] Govender, J., Veerasamy, D., y Noel, D. (2014). The Service Quality Experience of International Students: The Case of a Selected Higher Education Institution in South Africa. Mediterranean Journal of Social Sciences, 5(8) 465-473. Recuperado de https://doi. org/10.5901/mjss.2014.v5n8p465 


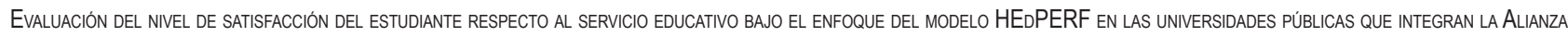
Estratégica de la Universidad Peruana y que implementaron el mecanismo de licenciamiento

[15] Hussein, B., Abou-Nassif, S., Airidi, M., Chamas, M., y Khachfe, H. (2017). Challenges of Implementation of ISO $9001: 2015$ in the Lebanese Higher Education Institutions. Journal of Resources Development and Management, 33, 41-51.

[16] Instituto Internacional de la UNESCO para la Educación Superior en América Latina y el Caribe. (2006). Informe sobre la Educación Superior en América Latina y el Caribe 20002005: La Metamorfosis de la educación superior. Caracas, Venezuela: Editorial Metropolis C.A.

[17] Jamieson, S. (2004). Likert Scale: How to (ab) Use Them. Medical Education 38(12), 1217 -1218. Recuperado de https://doi.org/10.1111/ j.1365-2929.2004.02012.x

[18] Juárez, F., López, E., y Villatoro, J. (2014). Estadística Inferencial Univariada. En: F. Juárez, J. López y V. Salinas (Eds.). Apuntes para la investigación en salud (161-282). México D.F., México: Editorial de la Universidad Nacional Autónoma de México.

[19] Ley $N^{\circ} 30220$ (2014, miércoles 9 de julio). Ley Universitaria. El Peruano Normas Legales: 527211-527233.

[20] Maldonado, M., y Moreta X. (2017). Medición de Calidad del Servicio mediante Modelo SERVPERF en Envasadora Ecuatoriana S.A. (Tesis de Maestría). Universidad de Guayaquil, Guayaquil.

[21] Murad, A., y Shastri, R. (2010). Implementation of Total Quality Management in Higher Education. Asian Journal of Business Management, 2(1), 9-16.

[22] Nadim, Z., y Al Hinai, A. (2016). Critical success factors of TQM in higher education institutions context. International Journal of Applied Sciences and Management, 1(2), 147-156.

[23] Nguyen, V., Nguyen, H., y Lam, P. (2014). A Research of Student's Satisfaction Towards Service Quality of Universities in Ho Chi Minh City. 2nd International Conference on Green Technology and Sustainable Development, Bangkok, Tailandia.

[24] Organización Internacional de Normalización. (2018). Organizaciones educativas - Sistemas de Gestión para Organizaciones Educativas Requisitos con orientación para su uso (ISO 21001:2018). Recuperado de https://www.iso. org/obp/ui\#iso:std:iso:21001:ed-1:v1:es
[25] Pontificia Universidad Católica de Chile. (2011). Sistema de aseguramiento de la calidad de la Educación Superior: aspectos críticos y desafíos de mejoramiento. Centro de Políticas Públicas UC. Recuperado de https://politicaspublicas.uc.cl/wp-content/ uploads/2015/02/sistema-de-aseguramientode-la-calidad-de-la-educacion-superior.pdf

[26] Purwanto, Y., Noor, I., y Kusumawati, A. (2020). Service Quality Measurement through Higher Education Performance (HEDPERF) The Case of an Indonesian Public University. Wacana Journal of Social and Humanity Studies, 23(1), 10-16. Recuperado de https://wacana.ub.ac.id/ index.php/wacana/article/view/676

[27] Resolución del Consejo Directivo $\mathrm{N}^{\circ}$ 011-2017-SUNEDU/CD. Otorgan Licencia Institucional a la Universidad Nacional Agraria La Molina, para ofrecer el servicio educativo superior universitario. Diario Oficial El Peruano (2017).

[28] Resolución del Consejo Directivo $\mathrm{N}^{\circ}$ 036-2018-SUNEDU/CD. Que otorga la licencia institucional a la Universidad Nacional Mayor de San Marcos, para ofrecer el servicio educativo superior universitario. Diario Oficial El Peruano (2018).

[29] Resolución del Consejo Directivo $\mathrm{N}^{\circ}$ 073-2017-SUNEDU/CD. Que otorga la licencia institucional a la Universidad Nacional de Ingeniería, para ofrecer el servicio educativo superior universitario. Diario Oficial El Peruano (2017).

[30] Schmatz, R., Wolf, G., y Landmann, M. (agosto de 2015). Students as customers: The Net Promoter Score as a measure of satisfaction and loyalty in higher education [artículo]. From here to there: Positioning higher education institutions - EAIR $37^{\text {th }}$ Annual Forum, Krems, Austria.

[31] Silva, D., Moraes, G., Makiya, I., y Cesar, F. (2017). Measurement of perceived service quality in higher education institutions: A review of HEdPERF scale use. Quality Assurance in Education, 25(4), 415-439. Recuperado de https://doi.org/10.1108/QAE-10-2016-0058

[32] Siti, O., Nor Alesha, M., Nur Shafini, M., y Khalid, M. (2020). The Effects of Service Quality Dimensions on Students' Satisfaction: HEdPERF Model Adoption. Jurnal Intelek. 15(1), 69-76. 
[33] Spilimbergo, A. (2009). Democracy and Foreign Education. Revisión Económica Americana, 99(1), 582-543. Recuperado de https://doi. org/10.1257/aer.99.1.528

[34] Sunanto, S., Taufiqurrahman, T., y Pangemanan, R. (2007). An Analysis of University Service Quality Gap and Student Satisfaction in Indonesia. The International Journal of Knowledge, Culture, and Change Management: Annual Review, 7(7), 1 -10. Recuperado de https://doi.org/10.18848/14479524/CGP/v07i07/50400
[35] Zineldin, M., y Vasicheva, V. (2014). The Implementation of TRM Philosophy and 5Qs Model in Higher Education - An Exploratory Investigation at a Swedish University. Nang Yan Business Journal, 1(1), 65-75. 


\section{Evaluation of the Student Satisfaction Level Regarding the Educational Service Under the Approach of the HEdPERF Model at Public Universities that Belong to the Strategic Alliance of Peruvian Universities and that Implemented the Licensing Mechanism ${ }^{1}$}

Rosa Carolina Alvarez TaY ${ }^{2}$

\begin{abstract}
The objective of this article was to establish the level of influence that the HEdPERF (Higher Education Performance) model has on student satisfaction, to establish the degree of influence that each of the six dimensions that make up the HEdPERF model have on student satisfaction, and to determine which of the six dimensions of the HEdPERF model presented an adequate level of perceived quality. The study sample consisted of 1191 students belonging to the three public universities that are members of the Alianza Estratégica de la Universidad Peruana (Strategic Alliance of Peruvian Universities). The research was non-experimental, at a correlational level, and non-parametric statistical tests were used for data analysis, such as the chi-square test, Spearman's correlation test and the Wilcoxon signedrank test.
\end{abstract}

Keywords: service quality; higher education; HEdPERF model; service quality assurance and student satisfaction.

\section{INTRODUCTION}

In its Report on Higher Education in Latin America and the Caribbean 2000-2005, the International Institute for Higher Education in Latin America and the Caribbean (IESALC) mention that, under the discourse of defending the autonomy of universities and before the total discrediting of the State as an effective provider of public services, it was decided that the students (clients) would assume responsibility for selecting the best educational options and that, in turn, the salaries of the graduates would be sanctioned by the market according to the value attributed to their degrees and institutions of origin (Instituto Internacional para la Educación Superior en América Latina y el Caribe, 2015).

In addition to this, Peru experienced a process of rapid increase of university enrollments during the last two decades, which, according to the Dirección General de Educación Superior Universitaria (General Directorate for Universities), was reflected in the figures from 1996 to 2012, where the growth rate was 2.5 times more than the previous years, as a result of the creation of 82 new universities (23 public and 59 private). This increase, added to little or almost no planning and the absence of sectoral policies for quality assurance, led the Peruvian State to recognize at the time that la educación superior universitaria no forma ciudadanos con altas capacidades para el ejercicio profesional competente y la producción de conocimiento de alto valor social [universities do not form citizens with high capacities for competent professional practice and the production of knowledge of high social value] (D.S. No. 016-2015-MINEDU, 2015).

\footnotetext{
A mechanism that allows the Peruvian State to ensure that institutions entering the market to provide higher education services comply with minimum quality requirements.

2 Degree in Science with a major in Business Management Engineering from Universidad Nacional Agraria La Molina, and specialist in Public Investment Project Management. Currently working as independent consultant (Lima, Peru). ORCID: https://orcid.org/0000-0002-6378-0142 Corresponding author: carolinaalvareztay@gmail.com
} 
Hence, the Peruvian State-faced with the urgent need to reform the university education system during the last decade (2009-2019)_, through the Ministry of Education and its attached public agencies, has deployed efforts through various mechanisms as a guarantee of educational quality whose center is the student (D.S. No. 016-2015-MINEDU, 2015); the most important are institutional licensing, accreditation of study programs and universities, and professional certification; the first being mandatory and the remaining two mechanisms, voluntary.

In this regard, the public universities that are members of the Strategic Alliance of Peruvian Universities $^{3}$ obtained their institutional licensing for up to ten years, which came into effect from the date of issuance in the following chronological order: Universidad Nacional Agraria La Molina (UNALM) in March 2017 through Resolución del Consejo Directivo No. 011-2017-SUNEDU/CD (2017); Universidad Nacional de Ingeniería (UNI) in November 2017 through Resolución del Consejo Directivo No. 073-2017-SUNEDU/CD (2017); and Universidad Nacional Mayor de San Marcos (UNMSM) in April 2018 through Resolución del Consejo Directivo No. 036-2018-SUNEDU/CD (2018). From the above, it is concluded that as of the date of this research, a period of time of approximately 2 to 3 years has elapsed since these universities obtained their licensing, a mechanism that evaluates Basic Quality Conditions (CBC, by its Spanish acronym).

In this sense, the problems that this research seeks to solve are stated below in the form of questions: (1) to what extent does the application of the HEdPERF (Higher Education Performance) Model, focused on the perceived quality of educational services, significantly influence student satisfaction?, (2) to what extent does each of the dimensions that make up the HEdPERF model significantly influence student satisfaction? and (3) which of the evaluated dimensions of the HEdPERF model present an adequate level of perceived quality? Hence, the objectives of this article are expressed as follows: (1) to establish the level of influence of the HEdPERF model, focused on the perceived quality of services, on student satisfaction, (2) to establish the degree of influence of each of the six dimensions that compose the HEdPERF model on student satisfaction, and (3) to determine which of the six evaluated dimensions of the HEdPERF model present an adequate level of perceived quality.

3 Signed on March 19, 2012, by the three national and historic universities (UNALM, UNMSM and UNI) with the purpose of contributing to the recovery, modernization and development of the public university in Peru.
The hypotheses of this work were formulated taking as a frame of reference the problems and objectives presented above; they are shown in detail below: (1) the perceived quality of the educational services of the HEdPERF model significantly influences student satisfaction, (2) the six dimensions that constitute the HEdPERF model significantly influence student satisfaction, and (3) the dimensions that constitute the HEdPERF model present an adequate level of perceived quality. Additionally, these hypotheses were contrasted with each of the public universities included in this research in order to compare them.

The main contribution that this article intends to make is directed towards the parties that constitute the Peruvian university education system, that is, students, those responsible for verifying compliance with all the Basic Quality Conditions (CBC), and the university authorities in charge of making academic and non-academic decisions, through the presentation, in qualitative and quantitative terms, of the perceived quality of educational services under the HEdPERF model approach and its degree of influence on student satisfaction. Additionally, the contribution of this study as new knowledge lies in the adaptation from English to Spanish of the HEdPERF model scale and its application to the reality of the public universities that are members of the Strategic Alliance of Peruvian Universities that have implemented the institutional licensing mechanism.

This study is based on the work of the following authors: Abdullah (2006), who states that the assessment of the quality level, as well as the understanding of how the different dimensions affect the overall quality of the educational service, will enable higher education institutions to efficiently design the service delivery process; Sunanto et al. (2007), who suggest that institutions should view students as their main customers and try to maximize their satisfaction based on the educational services provided; Spilimbergo (2009), who argues that the ability of universities to promote a quality institutional framework - by providing an environment conducive to democratic dialogue and exchange of ideas-allows for comprehensive training that includes the development of values and interpersonal skills that translate into greater social cohesion and democratic strengthening; Zineldin and Vasicheva (2014), who point out that measuring student perceptions of the quality of service offered by a university can reflect the overall student satisfaction level within the institution; Govender et al. (2014), who indicate that providing 
Evaluation of the Student Satisfaction level Regarding the Educational Service Under the Approach of the HEdPeRf Model at Public Universities that Belong to the Strategic Allance of Peruvian Universities and that Implemented the Licensing Mechanism

quality services is key for higher education institutions to differentiate themselves from their competitors and to ensure sustainability over a long time period; and finally, Nadim and AI Hinai (2016), who mention that quality in higher education institutions is one of the most significant aspects of knowledge creation, human resource development and social strength of any country.

In reference to other studies related to this article, researchers Abu et al. (2008) state that there is a strong positive relationship between perceived quality and student satisfaction; added to this, Gallifa and Batallé (2010) confirm that if the provision of educational services is not dynamic, with the passage of time the service is perceived as one of low quality; on the other hand, the authors Silva et al. (2017) systematically reviewed academic literature to evaluate the relevance of the HEdPERF model as a scale to measure service quality perceived by students in higher education institutions at an international level, and concluded that it is not appropriate to use a generic scale for all services, but a specific scale to quantitatively estimate the quality perceived in each one.

Finally, the theoretical framework that supports this research is structured in three main axes, as follows:

1. Quality management models applied to university-related institutions, for which a review of other similar articles was carried out, finding among the most outstanding models: (i) Total Quality Management (TQM) model, which is defined as a system of guarantees that was created to accommodate multiple stakeholders, as well as the diverse and changing roles of students in the educational process (Murad \& Shastri, 2010); (ii) Balanced Scorecard (BSC), which is a simple, logical, practical and verified management system, which allows an adequate response to modern challenges faced by higher education institutions (Eftimov et al., 2016); (iii) European Foundation for Quality Management (EFQM) model, described as a systematic evaluation carried out by an organization in all its areas, comparing them with a model of excellence that serves as a reference; this would allow top management to set improvement plans based on objective facts and a common vision of the goals to be achieved and the means to be employed (Del Campo et al., 2013); (iv) ISO 9001:2015, since, in a context of increasing enrollment rates, changing structure of society, different types of delivery of educational programs and largescale of job descriptions, the application of this standard is of great importance, as it provides a set of generic requirements for implementing a quality management system without regard to the activities performed by the organization (Hussein et al., 2017); (v) ISO 21001:2018, which mainly focuses on the critical and continuous evaluation of educational organizations in order to assess the degree of compliance with the requirements of students and other beneficiaries, so the global performance of the organization can be improved (Organización Internacional de Normalización, 2018); and (vi) modelo de sistema integrado de aseguramiento de calidad (comprehensive quality assurance system model), which is considered a good international practice and consists of the following elements: licensing, accreditation, academic auditing and information systems (Pontificia Universidad Católica de Chile, 2011). After the analysis of the aforementioned models, the comprehensive quality assurance system model was identified as the model that most resembles the Peruvian university context as a guarantee of educational quality.

2. Models used to study the quality of the university educational service. A thorough review of the academic literature on the main models used in university context was made, these included: (i) Net Promoter Score (NPS) model, which, according to authors Schmatz et al. (2015), consists of a question (the last question of the survey) that completely avoids terms such as "satisfaction" or "quality" and rather addresses something that goes even beyond customer satisfaction: loyalty (the willingness to make an investment or a personal sacrifice to strengthen a relationship), which allows predicting the actual behavior of customers; (ii) Kano model, defined by authors Arefi et al. (2012) as a tool widely used to perceive the voice of the customer in order to influence their own satisfaction; this model classifies the customer requirements in 6 categories: attractive elements, one-dimensional elements, must-be elements, indifferent elements, reverse elements and questionable elements; (iii) SERVQUAL model, conceptualized by authors Cañón and Rubio (2018) as a multiple response scale designed to understand customer expectations regarding a service received, through the evaluation of five dimensions: tangibility, reliability, responsiveness, assurance and empathy; (iv) SERVPERF model, which, according to authors Maldonado and Moreta (2018), focuses on customer perceptions of the service they receive, suppressing 
expectations, and which also provides diversity and variability according to the appropriate customer service; and (v) HEdPERF model, defined by its creator and author Abdullah (2005) as a scale that measures perceived quality specifically in the higher education sector, which is composed of 6 dimensions and 41 questions. In view of the above and appealing to the criteria of convenience and relevance for the present research, it was decided to apply the scale proposed by the HEdPERF model in the study.

3. Legal bases in the context of Peruvian university quality assurance, mainly comprised by: (1) The Nueva Ley Universitaria (Ley No. 30220, 2014), which implemented important changes such as the creation of the National Superintendence of Higher Education (SUNEDU), which is in charge of guaranteeing compliance with the Basic Quality Conditions (CBC) immersed in the institutional licensing process, and which specifies the accreditation process as voluntary with some exceptions; and (2) the Política de Aseguramiento de la Calidad de la Educación Superior Universitaria (Quality Assurance Policy for University), which establishes five principles (autonomy and responsible vice-chancellorship, student as the center, inclusion and equity, quality and academic excellence, and development of the country) and four pillars (reliable and timely information, encouragement to improve performance, accreditation for continuous improvement, and licensing as a guarantee of basic quality conditions) in the framework of the Peruvian university system.

\section{METHODOLOGY}

The research design was cross-sectional, non-experimental and correlational. It is described in detail below:

- It is cross-sectional, that is, the data were collected at a single moment (academic period 2019 - II) and its purpose was to describe the variables and analyze their incidence and correlation at a given moment.

- It is non-experimental, since the dependent variable (student satisfaction) in the study already occurred and it was not possible to manipulate it, that is, there was no direct control over that variable and its effects since they had already happened. By conducting a non-experimental research, the phenomenon was observed as it occurred in its natural context (public universities members of the Strategic Alliance of Peruvian Universities) for its subsequent analysis.

- It is correlational, since the relationships between the independent variables (perceived quality of educational services and subvariables) and the dependent variable (student satisfaction) were described, and their dimension and direction were established.

The unit of analysis was composed of enrolled undergraduate and graduate students, who belonged to the campuses that concentrate the largest number of study programs in the three public universities that are members of the Strategic Alliance of Peruvian Universities (See Table 1).

The characteristics of the unit of analysis regarding gender were $66 \%$ male, $31 \%$ female and $3 \%$ other. Regarding the age range of the students, $14 \%$ were between 14 and 18 years old, 58\% between 19 and 22 years old, $22 \%$ between 23 and 26 years old, and $6 \%$ between 27 and 58 years old. With respect to academic level, $96 \%$ were undergraduates and $4 \%$ were graduate students. In reference to the academic period, $54 \%$ stated that they were between the first and fourth cycle (first and second year), $33 \%$ were between the fifth and eighth cycle (third and fourth year) and 13\% were between the ninth and twelfth cycle (fifth and sixth year). Finally, with respect to the areas of knowledge, $71 \%$ belonged to the area of Engineering, $13 \%$ to the area of $\mathrm{Ba}-$ sic Sciences, $7 \%$ to the area of Humanities, Legal and Social Sciences, $6 \%$ to the area of Economics and Management Sciences, and $2 \%$ to the area of Health Sciences.

Table 1. Location of the Universities Considered in the Study.

\begin{tabular}{|l|l|l|}
\hline \multicolumn{1}{|c|}{ University } & \multicolumn{1}{c|}{ Address } & \multicolumn{1}{c|}{ District } \\
\hline Universidad Nacional Mayor de San Marcos & Calle German Amezaga No. 375 & Cercado de Lima \\
\hline Universidad Nacional Agraria La Molina & Avenida La Molina S/N & La Molina \\
\hline Universidad Nacional de Ingeniería & Avenida Túpac Amaru No. 210 & Rímac \\
\hline
\end{tabular}

Source: Prepared by the author. 
Evaluation of the Student Satisfaction Level Regarding the Educational Service Under the Approach of the HEdPerf Model at Public Universities that Belong to the Strategic Alliance of Peruvian Universities and that Implemented the Licensing Mechanism

The total sample size of the research, as shown in Table 2, was 1191 enrolled students and this was calculated using the historical values of the number of students enrolled between 2014 and 2018 in order to project the average number of students to be enrolled in 2019; subsequently, the formula to calculate the sample size for a finite population was used, with a confidence level of $95 \%$, and the following distribution was obtained for each university in the study:

The sample was random and proportionally stratified, applying the latter criterion according to each university. Other criteria were also applied, such as those detailed below:

Exclusion criterion: Undergraduate and graduate students who were not enrolled in the 2019-II academic period or who were not located, during the field study, within the campuses with the largest student population in the three public universities that are members of the Strategic Alliance of Peruvian Universities.

Elimination criteria: The surveys of those students who did not complete any of the items of the instrument or who duplicated their response in any of the requested items or who had unusual response patterns, such as choosing the same alternative in almost all the items, were eliminated.
The data was collected through a survey within the framework of the HEdPERF model, which consisted of 41 questions (items) that were brief, concise, and easy to understand. For the analysis and interpretation of the data, the MINITAB version 17 and SPSS version 25 statistical analysis and processing software packages were used. Other complementary software programs were also used for the extraction and visualization (tables and graphs) of the data, such as Microsoft Excel and Microsoft Power Point 2016.

\section{RESULTS}

Table 3 shows in detail the description of the 6 dimensions proposed by the HEdPERF model, whose correct understanding allowed the interpretation of the results obtained in this study and facilitated the subsequent discussion of the implications of this article.

As part of the preparatory acts to the application of the measurement instrument (survey) to the total sample, and to the contrast of the research hypotheses, a pilot test was developed with a subsample consisting of 53 students from the 3 public universities that are members of the Strategic Alliance of Peruvian Universities (17 from UNALM, 19 from UNI and 17 from UNMSM) with the purpose of evaluating the reliability and validity of the measurement instrument.

Table 2. Sample Size of the Study.

\begin{tabular}{|l|c|c|}
\hline University & Population Size & Sample Size \\
\hline Universidad Nacional Agraria La Molina & 13159 & 384 \\
\hline Universidad Nacional Mayor de San Marcos & 42787 & 392 \\
\hline Universidad Nacional de Ingeniería & 22888 & 415 \\
\hline Total & $\mathbf{7 8 ~ 8 3 4}$ & $\mathbf{1 1 9 1}$ \\
\hline
\end{tabular}

Source: Prepared by the author.

Table 3. Qualitative Description of the Dimensions of the HEdPERF Model.

\begin{tabular}{|l|l|}
\hline \multicolumn{1}{|c|}{ Dimension } & \multicolumn{1}{c|}{ Description } \\
\hline Academic aspects & Elements for which the teaching staff is fully responsible. \\
\hline Non-academic aspects & $\begin{array}{l}\text { Essential elements to allow that the student fulfills their study requirements. It refers to the tasks perfor- } \\
\text { med by the administrative staff. }\end{array}$ \\
\hline Reputation & $\begin{array}{l}\text { Elements that suggest the importance in higher education institutions of projecting a professional } \\
\text { image. }\end{array}$ \\
\hline Access & Elements related to accessibility, ease of contact, availability and convenience. \\
\hline Programs & $\begin{array}{l}\text { Elements that emphasize the importance of providing a wide range of highly reputable programs with } \\
\text { flexible structures and study plans. }\end{array}$ \\
\hline Understanding & $\begin{array}{l}\text { Elements related to the specific understanding the needs of the students in terms of counseling and } \\
\text { health. }\end{array}$ \\
\hline
\end{tabular}

Source: Adapted from Silva et al. (2017). 
The internal consistency of the items that make up each of the 6 dimensions of the HEdPERF model scale confirmed that the survey was reliable since its dimensions obtained Cronbach's alpha coefficients with values ranging between 0.780 and 0.924 (See Table 4).

The survey content was validated through the expert judgment technique. For this purpose, 3 university professors participated, these professors are specialists in the fields of psychometric test evaluation, postgraduate thesis evaluation and quality management of educational services. Consequently, the experts, through 5 evaluation criteria (congruence of the items, comprehensiveness of the content, wording of the items, clarity and precision, and relevance) expressed their opinions, which fluctuated between acceptable and excellent ratings (see Table 5), and gave the confidence to validate the content of the measurement instrument.

Kolmogorov Smirnov (SPSS version 25) and Anderson Darling (Minitab version 17) normality tests were used to verify the assumption of normality of the distribution of the data of variables (a) perceived quality of the educational service and its subvariables (dimensions of the HEdPERF model) and (b) student satisfaction. It was concluded that these present non-normal distributions, since they have a $p$-value less than 0.05 ( $\alpha$ value), with a confidence interval of $95 \%$ and, therefore, the research hypotheses were contrasted through non-parametric statistical tests (See Table 6).

Table 4. Results of Internal Consistency.

\begin{tabular}{|l|c|c|c|}
\hline Dimensions & Cronbach's Alpha & Number of items & Criteria \\
\hline Academic aspects & 0.893 & 9 & High reliability \\
\hline Non-academic aspects & 0.924 & 12 & Excellent reliability \\
\hline Reputation & 0.915 & 8 & Excellent reliability \\
\hline Access & 0.868 & 7 & High reliability \\
\hline Programs & 0.846 & 2 & High reliability \\
\hline Understanding & 0.780 & 2 & High reliability \\
\hline
\end{tabular}

Source: Prepared by the author.

Table 5. Results According to the Evaluation Criteria by the Expert Participants.

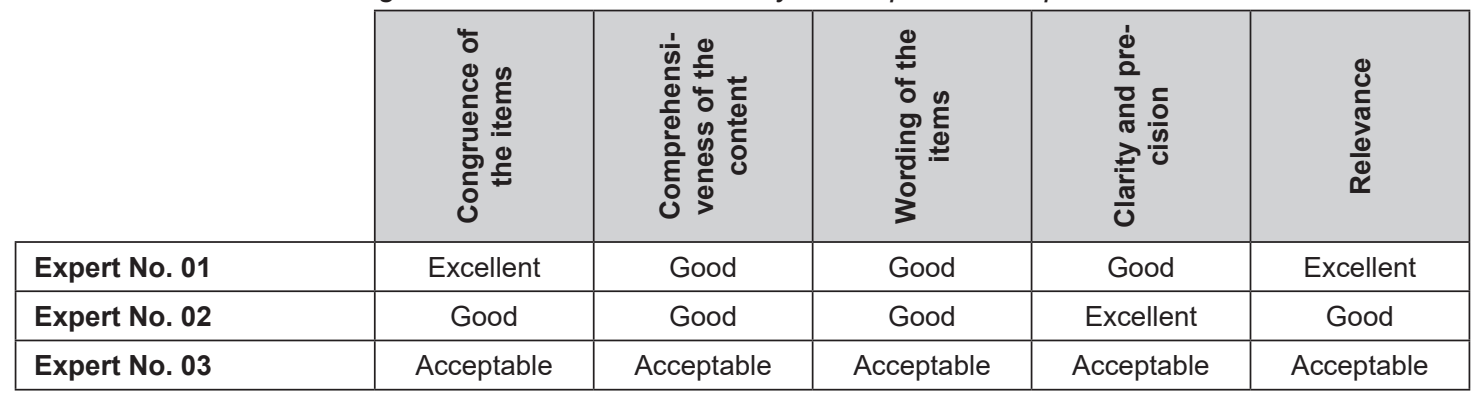

Source: Prepared by the author.

Table 6. Summary of Normality Tests.

\begin{tabular}{|l|c|c|c|}
\hline \multirow{2}{*}{\multicolumn{1}{|c|}{ Dimensions }} & \multicolumn{2}{c|}{ Kolmogorov - Smirnov } & Anderson Darling \\
\cline { 2 - 4 } & Test statistic & Asymptotic Significance (2-tailed) & $p$-value \\
\hline Academic aspects & 0.053 & 0.000 & $p$-value $<0.005$ \\
\hline Non-academic aspects & 0.051 & 0.000 & $p$-value $<0.005$ \\
\hline Reputation & 0.050 & 0.000 & $p$-value $<0.005$ \\
\hline Access & 0.050 & 0.000 & $p$-value $<0.005$ \\
\hline Programs & 0.124 & 0.000 & $p$-value $<0.005$ \\
\hline Understanding & 0.104 & 0.000 & $p$-value $<0.005$ \\
\hline Service satisfaction & 0.337 & 0.000 & $p$-value $<0.005$ \\
\hline
\end{tabular}

Source: Prepared by the author. 
Evaluation of the Student Satisfaction Level Regarding the Educational Service Under the Approach of the HEdPerf Model at Public Universities that Belong to the Strategic Alliance of Peruvian Universities and that Implemented the Licensing Mechanism

The following nonparametric statistical tests were used to contrast the hypotheses in the article: (1) the Chi-squared test, which allows determining the association or independence of two qualitative variables through the use of contingency tables where categorical data are summarized in rows and columns; (2) the Wilcoxon signed-ranks test, which according to authors Juárez et al. (2014), is used to compare the median (central position in a set of ordered data) of two related samples and determine whether there are differences between them; and (3) Spearman's correlation test, which allows to know the degree of association between two variables through Spearman's Rho, which in turn determines the dependence or independence of two random variables, as well as how strong the relationship between the variables is and whether the relationship between these variables is positive, null or negative (Elorza \& Medina, 1999).

The results for the first research hypothesis are shown in Table 7, where it is observed that students who rated the perceived quality of the services as "high" were the most satisfied with these educational services.

Additionally, for the first research hypothesis, Table 8 (contingency table) - where the results of the chisquare test have a confidence level of $95 \%$ - shows that the calculated value of Pearson's chi-square (242.419) was greater than the critical value of the chi-square (9.4877). Moreover, the significance coefficient $(p=0.000)$ resulted to be less than 0.05 ( $p$ $<0.05$ ), which allowed us to accept the research hypothesis.

Therefore, it can be stated that the perceived quality of educational services of HEdPERF model influences or impacts the satisfaction of students belonging to the three public universities that are members of the Strategic Alliance of Peruvian Universities.

Similarly, regarding the contrast of the second research hypothesis, which sought to prove that each of the dimensions of the HEdPERF model influences student satisfaction, it was verified through the non-parametric chi-square test that these six dimensions influenced student satisfaction (see Table 9). On the other hand, the influence of the six dimensions on student satisfaction was tested according to each alma mater (UNALM, UNMSM and UNI), concluding that there is a dependency relationship.

After verifying in the second hypothesis that there was dependence between the variables perceived quality of educational services and student satisfaction, as well as dependence between each of the six dimensions of the HEdPERF model and variable student satisfaction by means of the chi-square test, the Spearman correlation non-parametric test was used to verify the type of association, whose results showed a moderate positive correlation for

Table 7. Level of Influence of the Perceived Quality of Educational Services and Student Satisfaction.

\begin{tabular}{|c|c|c|c|c|c|c|}
\hline & & & \multirow{2}{*}{\multicolumn{3}{|c|}{ Student Satisfaction }} & \multirow[b]{3}{*}{ Total } \\
\hline & & & & & & \\
\hline & & & Disappointed & Indifferent & Delighted & \\
\hline \multirow{6}{*}{ 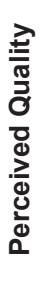 } & \multirow[t]{2}{*}{ Low } & Observed & 18.0 & 12.0 & 5.0 & 35.0 \\
\hline & & Expected & 4.7 & 8.8 & 21.5 & 35.0 \\
\hline & \multirow[t]{2}{*}{ Average } & Observed & 70.0 & 96.0 & 50.0 & 216.0 \\
\hline & & Expected & 28.8 & 54.6 & 132.6 & 216.0 \\
\hline & \multirow[t]{2}{*}{ High } & Observed & 71.0 & 193.0 & 676.0 & 940.0 \\
\hline & & Expected & 125.5 & 237.6 & 576.9 & 940.0 \\
\hline & \multirow[t]{2}{*}{ Total } & Observed & 159.0 & 301.0 & 731.0 & 1191.0 \\
\hline & & Expected & 159.0 & 301.0 & 731.0 & 1191.0 \\
\hline
\end{tabular}

Source: Prepared by the author.

Table 8. Chi-Square Test for Quality Perception of Educational Services and Student Satisfaction.

\begin{tabular}{|l|c|c|c|}
\hline \multicolumn{1}{|c|}{ Statistics } & Value & Df & Asymptotic Significance (2-tailed) \\
\hline Pearson's chi-square test & 242.419 & 4 & 0.000 \\
\hline Likelihood-ratio test & 229.4815 & 4 & 0.000 \\
\hline N of valid cases & 1191 & & \\
\hline
\end{tabular}

Source: Prepared by the author. 
the dimensions academic aspects, non-academic aspects, programs and understanding; that is to say, their values were between 0.11 and 0.50 ; while, for the dimensions reputation and access, a considerable positive correlation was found to exist with values between 0.51 and 0.75 (see Table 10).

The same analysis was carried out per university. The results showed that for UNALM students, the dimensions with a moderate positive correlation were academic aspects, non-academic aspects, reputation, programs and understanding; while the only dimension that obtained a considerable positive correlation was access. In the case of UNMSM, the academic aspects and understanding dimensions had a moderate positive correlation, while the other dimensions obtained a considerable positive correlation. Finally, UNI obtained a moderate positive correlation for the six dimensions evaluated.

Similarly, to test the third hypothesis of the research-regarding the identification of which of the six evaluated dimensions of the HEdPERF model presented a high level of perceived quality, that is, between the categories or ratings of "Very Good" and "Excellent"- the Wilcoxon non-parametric statistical test was applied, comparing the calculated median with a hypothetical median. It was found that 4 of the 6 dimensions evaluated (academic aspects, non-academic aspects, access and understanding) do not have a high level of perceived quality; in contrast, the remaining 2 dimensions (reputation and programs) obtained high levels of perceived quality (see Table 11).

Table 9. Chi-Square Test of the Six Dimensions of HEdPERF Model and Student Satisfaction Variable.

\begin{tabular}{|c|l|c|l|}
\hline No. & $\begin{array}{c}\text { Dimensions of the HEd- } \\
\text { PERF model }\end{array}$ & $\begin{array}{c}\boldsymbol{\alpha} \text { value versus } \\
\boldsymbol{p} \text {-value }\end{array}$ & \multicolumn{1}{|c|}{ Result of the chi-square test } \\
\hline 1 & Academic aspects & $0.000<0.05$ & Dimension "academic aspects" influences student satisfaction \\
\hline 2 & Non-academic aspects & $0.000<0.05$ & Dimension "non-academic aspects" influences student satisfaction \\
\hline 3 & Reputation & $0.000<0.05$ & Dimension "reputation" influences student satisfaction \\
\hline 4 & Access & $0.000<0.05$ & Dimension "access" influences student satisfaction \\
\hline 5 & Programs & $0.000<0.05$ & Dimension "programs" influences student satisfaction \\
\hline 6 & Understanding & $0.000<0.05$ & Dimension "understanding" influences student satisfaction \\
\hline
\end{tabular}

Source: Prepared by the author.

Table 10. Spearman's Correlation Test of the Six Dimensions of the HEdPERF Model and Student Satisfaction Variable.

\begin{tabular}{|c|l|c|l|}
\hline No. & Dimensions of the HEdPERF model & Correlation coefficient (Rho) & Type of correlation \\
\hline 1 & Academic aspects & 0.411 & Medium or moderate positive correlation \\
\hline 2 & Non-academic aspects & 0.487 & Medium or moderate positive correlation \\
\hline 3 & Reputation & 0.528 & Significant positive correlation \\
\hline 4 & Access & 0.526 & Significant positive correlation \\
\hline 5 & Programs & 0.464 & Medium or moderate positive correlation \\
\hline 6 & Understanding & 0.478 & Medium or moderate positive correlation \\
\hline
\end{tabular}

Source: Prepared by the author.

Table 11. Wilcoxon Signed-Rank Test of the Six Dimensions with Respect to a Hypothetical Median.

\begin{tabular}{|c|l|l|l|}
\hline No. & $\begin{array}{c}\text { Dimensions of the } \\
\text { HEdPERF model }\end{array}$ & $\begin{array}{c}\mathbf{p} \text {-value versus } \\
\boldsymbol{\alpha} \text { value }\end{array}$ & \multicolumn{1}{|c|}{ Results of the Wilcoxon signed-rank test } \\
\hline 1 & Academic aspects & $0.9730>0.05$ & The dimension "academic aspects" does not have a high level of perceived quality. \\
\hline 2 & Non-academic aspects & $1.0000>0.05$ & $\begin{array}{l}\text { The dimension "non-academic aspects" does not have a high level of perceived } \\
\text { quality. }\end{array}$ \\
\hline 3 & Reputation & $0.0000<0.05$ & The dimension "reputation" has a high level of perceived quality. \\
\hline 4 & Access & $1.0000>0.05$ & The dimension "access" does not have a high level of perceived quality. \\
\hline 5 & Programs & $0.0000<0.05$ & The dimension "programs" has a high level of perceived quality \\
\hline 6 & Understanding & $0.1000>0.05$ & The dimension "understanding" does not have a high level of perceived quality. \\
\hline
\end{tabular}

Source: Prepared by the author. 
Evaluation of the Student Satisfaction Level Regarding the Educational Service Under the Approach of the HEdPerf Model at Public Universities that Belong to the Strategic Allance of Peruvian Universities and that Implemented the Licensing Mechanism

It was also considered convenient to evaluate the third research hypothesis specifically according to the university of origin of the students. It was obtained that for UNALM, the dimensions that presented a high level of perceived quality were academic aspects, reputation, access, programs and understanding; for UNMSM, only the dimension programs obtained a high level of perceived quality; finally, in the case of UNI, none of the dimensions obtained adequate levels of perceived quality.

\section{DISCUSSION}

The internal consistency of the items that make up each of the six dimensions of the HEdPERF scale showed that the survey is reliable since its dimensions obtained Cronbach's alpha coefficients with values ranging between 0.780 and 0.924 . The latter coincides with the results obtained by authors $A b$ dullah (2006) and Brochado (2009), who concluded that the HEdPERF scale is the one that best fits the context of higher education in comparison with other "classic" measurement instruments such as SERVQUAL and SERVPERF.

In reference to the verification of the normality of the distribution of the data of the variables perceived quality of the educational service, dimensions and student satisfaction, through the Kolmogorov Smirnov and Anderson Darling normality tests, it was concluded that they present non-normal distributions. For this reason, it was considered convenient to apply non-parametric statistics to test the three research hypotheses of this article. It was confirmed what was pointed out by the author Jamieson (2004), who indicates that only non-parametric statistics should be used for the analysis of the Likert scale data.

Through the first research hypothesis, it was shown that there is a significant and positive relationship between the perceived quality of educational services proposed by the HEdPERF model and student satisfaction, as evidenced by previous studies (Nguyen et al., 2014; Banahene et al., 2018; Siti et al., 2020). On the other hand, regarding the second research hypothesis, it was also demonstrated that there is a significant and positive relationship between each of the six dimensions and student satisfaction. Finally, for the third research hypothesis, it was proven that the dimensions possessing a high level of perceived quality were "reputation" and "programs"; the latter contributes to the results obtained by other authors who posed similar research hypotheses (Ali et al. 2016; Purwanto et al., 2020).
In relation to the generalization of the research, based on the results of the total sample (1191 students) and the results stratified per university (384 students from UNALM, 392 students from UNMSM and 415 students from UNI), it can be affirmed that depending on the context in which the scale is applied, it is possible to find differences between the six dimensions that make up the HEdPERF model and their degree of influence on student satisfaction, as well as between the levels of quality perceived by each of the dimensions of the HEdPERF model.

This research is a contribution to theoretical knowledge in the framework of the various models that have been applied to measure the quality of service in higher education institutions, because, for the first time, the scale proposed by the HEdPERF model was applied in a context of Peruvian public universities (Universidad Nacional Mayor de San Marcos, Universidad Nacional Agraria La Molina and Universidad Nacional de Ingeniería). In addition, the originality of this study lies in the translation from English to Spanish of the measurement instrument mentioned, as well as the contribution of new dimensions to evaluate the perceived quality of educational services, such as: academic aspects, non-academic aspects, reputation, access, programs and understanding; according to its author and creator Abdullah (2005). Finally, this research makes a contribution to the systemic review of the academic literature on educational quality management models, as well as the main scales used to study the quality of educational services in higher education institutions and the current regulations regarding quality assurance in Peruvian university higher education.

On the other hand, the practical implications of the study made it possible to show in qualitative and quantitative terms the quality perceived in educational services under the HEdPERF model approach and its degree of influence on student satisfaction in a context subsequent to the implementation of the institutional licensing mechanism.

\section{CONCLUSIONS}

- The HEdPERF model scale has proven to be a valid and reliable instrument due to its application to undergraduate and graduate students of the public universities that are members of the Strategic Alliance of Peruvian Universities, so it can be used in future research studies. 
- The first research hypothesis was proved, and it was concluded that the perceived quality proposed by the HEdPERF model significantly influences student satisfaction, for which non-parametric statistical tests such as chi-square and Spearman's correlation were used.

- The second research hypothesis was proved, so it can be affirmed that the level of perceived quality in each of the six dimensions of the HEdPERF model significantly influences student satisfaction, for which the nonparametric statistical tests chi-square and Spearman's correlation were used.

- The third research hypothesis was tested by the Wilcoxon signed-rank test, determining the levels of perceived quality for each of the dimensions of the HEdPERF model; obtaining that four of the six dimensions evaluated (academic aspects, non-academic aspects, access and understanding) do not have a high level of perceived quality, while the remaining two dimensions (reputation and programs) obtained high levels of perceived quality.

- The Wilcoxon test analysis was performed for each of the universities included in the scope of this research and it was found that for UNALM, the dimensions that presented a high level of perceived quality were academic aspects, reputation, access, programs and understanding; for UNMSM, only the dimension programs obtained a high level of perceived quality; and for UNI, none of the dimensions obtained high levels of perceived quality. From the above, it can be concluded that there are differences in the levels of perceived quality among the dimensions according to each university.

\section{REFERENCES}

[1] Abdullah, F. (2005). HEdPERF versus SERVPERF: The quest for ideal measuring instrument of service quality in higher education sector. Quality Assurance in Education, 13(4), 305-328. Retrieved from https://doi. org/10.1108/09684880510626584

[2] Abdullah, F. (2006). The development of HEdPERF: a new measuring instrument of service quality for the higher education sector. Internacional Journal of Consumer Studies, 30(6), 569-581. Retrieved from https://doi. org/10.1111/j.1470-6431.2005.00480.x
[3] Abu Hasan, H., Illias, A., Rahman, R., \& Razack, M. (2008). Service Quality and Student Satisfaction: A Case Study at Private Higher Education Institutions. International Business Research, 1(3),163-175. Retrieved from https:// doi.org/10.5539/ibr.v1n3p163

[4] Ali, F., Zhou, Y., Hussain, K., Nair, P., \& Ravagan, N. (2016). Does higher education service quality effect student satisfaction, image and loyalty? A study of international students in Malaysian public universities. Quality Assurance in Education, 24(1), 70-94. Retrieved from https://doi.org/10.1108/QAE02-2014-0008

[5] Arefi, M., Heidari, M., Morkani, G., \& Zandi, K. (2012). Application of Kano Model in Higher Education Quality Improvement: Study Master's Degree Program of Educational Psychology in State Universities of Tehran. World Applied Sciences Journal, 17 (3), 347353. Retrieved from https://www.idosi.org/wasj/ wasj17(3)12/12.pdf

[6] Banahene, S., Kraa, J., \& Kasu, P. (2018). Impact of HEdPERF on Students' Satisfaction and Academic Performance in Ghanaian Universities; Mediating Role of Attitude towards Learning. Open Journal of Social Sciences, 6(5), 96-119. Retrieved from https://doi. org/10.4236/jss.2018.65009

[7] Brochado, A. (2009). Comparing alternative instruments to measure service quality in higher education. Quality Assurance in Education, 17(2), $174-190$. Retrieved from https://doi. org/10.1108/09684880910951381

[8] Cañón, A. , \& Rubio, D. (2018). Importancia de la utilización del modelo SERVQUAL a partir de una revisión sistemática de la literatura en el periodo 2010-2016. (Trabajo de grado - especialización). Fundación Universitaria de Ciencias de la Salud, Bogotá. Retrieved from https://repositorio.fucsalud.edu.co/ handle/001/669

[9] D.S. No. 016-2015-MINEDU. Aprueban la política de aseguramiento de la calidad de la educación superior universitaria. Diaria Oficial El Peruano (2015).

[10] Del Campo, M., Ferreiro, F, \& Camino, M. (July, 2013). Una aplicación del modelo EFQM a la educación superior. XXII Jornadas de la Asociación de la Economía de la Educación. Retrieved from http://2013.economicsofeducation. 
Evaluation of the Student Satisfaction Level Regarding the Educational Service Under the Approach of the HEdPerf Model at Public Universities that Belong to the Strategic Allance of Peruvian Universities and that Implemented the Licensing Mechanism

com/user/pdfsesiones/138. pdf?PHPSESSID=evsluk1nq0k6noc2eipo8tInt7

[11] Eftimov, L., Trpeski, P., Gockov, G., \& Vasileva, V. (2016). Designing a Balanced Scorecard as Strategic Management System for Higher Education Institutions: A Case Study in Macedonia. Ekonomika, Journal for Economic Theory and Practice and Social Issues, 62(2), 29-48. Retrieved from https://doi.org/10.22004/ ag.econ. 288842

[12] Elorza, H., \& Medina, J. (1999). Estadística para las ciencias sociales y del comportamiento. México D. F., Mexico: Oxford University.

[13] Gallifa, J., \& Batallé, P. (2010). Percepción de los estudiantes sobre la calidad del servicio en un sistema de educación superior con varios campus en España. Revista de Aseguramiento de la Calidad en la Educación, 18(2), 156 - 170. Retrieved from https://doi. org/10.1108/09684881011035367

[14] Govender, J., Veerasamy, D., \& Noel, D. (2014). The Service Quality Experience of International Students: The Case of a Selected Higher Education Institution in South Africa. Mediterranean Journal of Social Sciences, 5(8) ,465-473. Retrieved from https://doi. org/10.5901/mjss.2014.v5n8p465

[15] Hussein, B., Abou-Nassif, S., Airidi, M., Chamas, M. \&, Khachfe, H. (2017). Challenges of Implementation of ISO 9001:2015 in the Lebanese Higher Education Institutions. Journal of Resources Development and Management, 33, 41-51.

[16] Instituto Internacional de la UNESCO para la Educación Superior en América Latina y el Caribe. (2006). Informe sobre la Educación Superior en América Latina y el Caribe 20002005: La Metamorfosis de la educación superior. Caracas, Venezuela: Editorial Metropolis C.A.

[17] Jamieson, S. (2004). Likert Scale: How to (ab) Use Them. Medical Education 38(12), 1217 -1218. Retrieved from https://doi.org/10.1111/ j.1365-2929.2004.02012.x

[18] Juárez, F., López, E., \& Villatoro, J. (2014). Estadística Inferencial Univariada. In: F. Juárez, J. López, \& V. Salinas (Eds.). Apuntes para la investigación en salud (161-282). México D.F., Mexico: Editorial de la Universidad Nacional Autónoma de México.

[19] Ley No. 30220 (July 9, 2014). Ley Universitaria. El Peruano Normas Legales: 527211-527233.
[20] Maldonado, M., \& Moreta X. (2017). Medición de Calidad del Servicio mediante Modelo SERPERF en Envasadora Ecuatoriana S.A. (Master thesis). Universidad de Guayaquil, Guayaquil.

[21] Murad,A., \& Shastri, R. (2010). Implementation of Total Quality Management in Higher Education. Asian Journal of Business Management, 2(1), 9-16.

[22] Nadim, Z., \& Al Hinai, A. (2016). Critical success factors of TQM in higher education institutions context. International Journal of Applied Sciences and Management, 1(2), 147-156.

[23] Nguyen, V., Nguyen, H., \& Lam, P. (2014). A Research of Student's Satisfaction Towards Service Quality of Universities in Ho Chi Minh City. 2nd International Conference on Green Technology and Sustainable Development, Bangkok, Thailand.

[24] Organización Internacional de Normalización. (2018). Organizaciones educativas - Sistemas de Gestión para Organizaciones Educativas Requisitos con orientación para su uso (ISO 21001:2018). Retrieved from https://www.iso. org/obp/ui\#iso:std:iso:21001:ed-1:v1:es

[25] Pontificia Universidad Católica de Chile. (2011). Sistema de aseguramiento de la calidad de la Educación Superior: aspectos críticos y desafíos de mejoramiento. Centro de Políticas Públicas UC. Retrieved from https://politicaspublicas. uc.cl/wp-content/uploads/2015/02/sistema-deaseguramiento-de-la-calidad-de-la-educacionsuperior.pdf

[26] Purwanto, Y., Noor, I., \& Kusumawati, A. (2020). Service Quality Measurement through Higher Education Performance (HEDPERF) The Case of an Indonesian Public University. Wacana Journal of Social and Humanity Studies, 23(1), 10-16. Retrieved from https://wacana.ub.ac.id/ index.php/wacana/article/view/676

[27] Resolución del Consejo Directivo No. 011-2017-SUNEDU/CD. Otorgan Licencia Institucional a la Universidad Nacional Agraria La Molina, para ofrecer el servicio educativo superior universitario. Diario Oficial El Peruano (2017).

[28] Resolución del Consejo Directivo No. 036-2018-SUNEDU/CD. Que otorga la licencia institucional a la Universidad Nacional Mayor de San Marcos, para ofrecer el servicio educativo superior universitario. Diario Oficial El Peruano (2018). 
[29] Resolución del Consejo Directivo No. 073-2017-SUNEDU/CD. Que otorga la licencia institucional a la Universidad Nacional de Ingeniería, para ofrecer el servicio educativo superior universitario. Diario Oficial El Peruano (2017).

[30] Schmatz, R., Wolf, G., \& Landmann, M. (August, 2015). Students as customers: The Net Promoter Score as a measure of satisfaction and loyalty in higher education [artículo]. From here to there: Positioning higher education institutions - EAIR $37^{\text {th }}$ Annual Forum, Krems, Austria.

[31] Silva, D., Moraes, G., Makiya, I., \& Cesar, F. (2017). Measurement of perceived service quality in higher education institutions: A review of HEdPERF scale use. Quality Assurance in Education, 25(4), 415-439. Retrieved from https://doi.org/10.1108/QAE-10-2016-0058

[32] Siti, O., Nor Alesha, M., Nur Shafini, M., \& Khalid, M. (2020). The Effects of Service Quality Dimensions on Students' Satisfaction: HEdPERF Model Adoption. Jurnal Intelek. 15(1), 69-76.
[33] Spilimbergo, A. (2009). Democracy and Foreign Education. Revisión Económica Americana, 99(1), 582-543. Retrieved from https://doi. org/10.1257/aer.99.1.528

[34] Sunanto, S., Taufiqurrahman, T., \& Pangemanan, R. (2007). An Analysis of University Service Quality Gap and Student Satisfaction in Indonesia. The International Journal of Knowledge, Culture, and Change Management: Annual Review, 7(7), 1 -10. Retrieved from https://doi.org/10.18848/14479524/CGP/v07i07/50400

[35] Zineldin, M., \& Vasicheva, V. (2014). The Implementation of TRM Philosophy and 5Qs Model in Higher Education - An Exploratory Investigation at a Swedish University. Nang Yan Business Journal, 1(1), 65-75. 
